Research Article

\title{
Cavitation Analysis in Centrifugal Pumps Based on Vibration Bispectrum and Transfer Learning
}

\author{
Ali Hajnayeb \\ Mechanical Engineering Department, Shahid Chamran University of Ahvaz, Ahvaz, Iran \\ Correspondence should be addressed to Ali Hajnayeb; a.nayeb@scu.ac.ir
}

Received 6 November 2021; Accepted 22 November 2021; Published 2 December 2021

Academic Editor: Yi Qin

Copyright (C) 2021 Ali Hajnayeb. This is an open access article distributed under the Creative Commons Attribution License, which permits unrestricted use, distribution, and reproduction in any medium, provided the original work is properly cited.

\begin{abstract}
Detection of cavitation in centrifugal pumps is critical in their condition monitoring. In order to detect cavitation more accurately and confidently, more advanced signal processing techniques are needed. For the classification of a pump conditions based on the outputs of these techniques, advanced machine learning techniques are needed. In this research, an automatic system for cavitation detection is proposed based on machine learning. Bispectral analysis is used for analyzing the vibration signals. The resulting bispectrum images are given to convolutional neural networks (CNNs) as inputs. The CNNs are a pretrained AlexNet and a pretrained GoogleNet, which are used in this application through transfer learning. On the contrary, a laboratory test setup is used for generating controlled cavitation in a centrifugal pump. The suggested algorithm is implemented on the vibration dataset acquired from the laboratory pump test setup. The results show that the cavitation state of the pump can be detected accurately using this system without any need to image processing or feature extraction.
\end{abstract}

\section{Introduction}

Vibration analysis combined with machine learning has been one of the effective techniques for analyzing cavitation in centrifugal pumps as well as other rotating machinery. Even recently, detecting cavitation in centrifugal pumps using simple vibration techniques has been an intriguing research topic [1-6]. Vibration analysis has been also applied to extract cavitation-related features, which can be used in classifiers for fault diagnosis. For that purpose, different types of classifiers and optimizers have been used in order to improve the calculation speed and diagnosis accuracy [7-11]. As the signal-based methods [12-15] for machinery diagnosis, as well as the model-based methods [16-19], have been applied, the research on both newer vibration analysis techniques and more advanced machine learning tools for accurate cavitation analysis is still continued.

One of the vibration analysis techniques especially for cavitation detection is bispectrum analysis. It has been used for fault diagnosis of rotating and reciprocating systems [20-23]. However, it has been applied in fault diagnosis of pumps only in limited number of studies despite its promising results. Hamomd et al. [24] used the modulation signal bispectrum in order to diagnose a number of faults including bearing and vane blockage in centrifugal pumps. Through experiments, they showed the effectiveness of the features extracted from the bispectrum of the signals. They used the low-frequency part of the vibration signal and proved its effectiveness in experiments. Li et al. [25] extracted bispectral entropy features from the vibration signal of a pump for fault prognosis. The results showed acceptable accuracy in the predictions of the pump's remaining useful life. Sandhya et al. [26] introduced and used a phase coupling index through bispectrum to detect the onset of cavitation of a ship propeller. They experimentally measured the noise data from a ship propeller and showed the effectiveness of their proposed method.

Several years ago, the initial machine learning tools opened a door to automatic fault detection of machinery without relying on experts' knowledge. At first, they seemed to be limited and not able to handle a large amount of data or complicated problems; however, the deep neural networks compensated for the shortcomings of those initial systems. Among all the deep neural networks, convolutional 
networks are one of the most applied ones. Conventional machine learning has been used for condition monitoring of rotating or reciprocating machines and components, such as pumps and compressors [27, 28], gears [29,30], ball bearings and engines [31-33], and even combinations of systems or combined faults [34]. These initial machine learning techniques had limited learning capability and the input size was limited in practice. Therefore, effective features had to be extracted from the vibration signal, and in the case of large feature vectors, a feature selection algorithm was also needed $[29,35,36]$. This limitation has made it difficult to use the output data of vibration analysis methods if they would produce large data such as images.

After the introduction of deep neural networks, which can handle large input data samples, most of the research in the field of machine learning-based vibration diagnosis is focused on them. They can also extract the necessary feature for classifications. Guo et al. [37] used a convolutional neural network and continuous wavelet transform of vibration signals as its input data in order to diagnose rotating machinery faults. Wang et al. [28] combined the vibration signals obtained simultaneously from multiple sensors and represented the combinations as images. Then, they used a CNN to recognize the faults. Pham et al. [38] applied vibration spectrums of bearings and a CNN to diagnose the faults of bearings under variable-shaft-speed conditions. Chen and Li [39] used a combination of a sparse autoencoder and a deep belief network in order to apply the vibration data of different vibration sensors for fault diagnosis. Despite the advantages the deep neural networks have over conventional machine learning methods, they still need a large amount of training data.

Transfer learning, which is a technique in which a model trained for a machine learning task is transferred completely or partially to another machine learning task, has been used in machinery diagnosis too. By using this technique, less training data in the final machine learning task would be needed even for complicated deep neural networks, and the trained network can be used for other working conditions of a machine. Qian et al. [40] proposed a novel transfer learning method in order to diagnose machinery faults. They applied the method to an experimental vibration dataset of bearings and a gearbox and compared the results with other techniques. Sandeep et al. [41] introduced a classifier based on a CNN and transfer learning in order to diagnose different faults of bearings under variable working conditions using kurtograms as inputs to the CNN. In a recent study, Grover and Turk [21] generated the bispectrum images from vibration signals of a bearing in cases of different fault types. Then, they used four initially trained deep neural networks including AlexNet, GoogLeNet, ResNet, and VGGNet for transfer learning. A comparative study was conducted on a benchmark vibration dataset. Dong et al. [42] processed the vibrations of machinery using wavelet transform. Then, they selected the best features and pretrained a deep autoencoder to be used for the final fault diagnosis task through transfer learning.

Based on the reviewed literature, it can be observed that bispectrum has the potential to be used in cavitation detection. Moreover, the bispectrum diagrams can be saved as images, which can be analyzed in deep neural networks for a high-accuracy classification. Because of limited studies on applying transfer learning and bispectrum analysis for detecting cavitation in pumps, this research is focused on that topic. Therefore, the vibration data of a centrifugal pump under the normal operating condition and also after the onset of cavitation are analyzed using bispectral analysis. The generated bispectrum diagrams are then used as the input to two pretrained deep neural networks for image processing. The onset of cavitation and also the severity level were detected using deep transfer learning. The test results show that the final neural network can accurately detect the occurrence of cavitation in the pump despite the limited number of data used for training. The results of cavitation intensity detection were also promising despite the difficulty of the task.

\section{Theory}

In this section, a brief review of the theoretical concepts of bispectrum and transfer learning is provided.

2.1. Bispectrum. Bispectrum is the Fourier transform of the third cumulant of a signal. It can present the coupling between two frequency components. It is defined for a signal, $x(t)$, as [43]

$$
B_{x}\left(f_{1}, f_{2}\right)=E\left[X\left(f_{1}\right) X\left(f_{2}\right) X^{*}\left(f_{1}+f_{2}\right)\right],
$$

where $X(f)$ and $X^{*}(f)$ denote the Fourier transform of the signal and its complex conjugate, respectively. In other words, it is a two-dimensional Fourier transform with an autocorrelation of third order [44]. Bispectrum is affected by the nonlinearities of the analyzed signal. It can detect the non-Gaussianity and also the correlation between frequency components of a signal. Especially, the phase coupling can be investigated between two frequency components.

Because of the nature of the cavitation signal, bispectrum can extract effective features from the raw vibration signal. In this research, the vibration signal of the pump is analyzed using bispectral analysis, and the resulting bispectrum, which contains information about the health conditions, is saved as RGB images in order to be fed into the deep neural network, later.

2.2. Transfer Learning. In transfer learning, the knowledge learned in a task, called the source task, is transferred to another task, called the target task. Through this transfer, the generalization of the target task improves by using the available knowledge of the source task. In the case of using a CNN, transfer learning means training a CNN based on the source task and transferring the convolutional layers of the trained CNN to the target task, where the weights of the fully connected layers are determined through another training process using the dataset of the target task. It is known that the convolutional layers are used for extraction of the features and are usually applicable identically for different problems, while the fully connected layers perform the desired final task and are supposed to 
be uniquely trained for each specific task. Therefore, a powerful feature extraction part can be used for a different problem if the weights of the fully connected layers are tuned for that. Because of the complicated structures of CNNs, this approach saves significant amounts of time and effort in the training process.

In this study, AlexNet, which is a well-known pretrained $\mathrm{CNN}$ for image classification is used. As discussed earlier, except for the fully connected layers, all the layers are transferred to be used in this target task, which is the classification of the bispectrum images. The complete structure of the CNN used in this research based on AlexNet is shown in Table 1. The fully connected layers of AlexNet are substituted by two new fully connected layers. The first one has 5 neurons and the second one has 2 neurons. These layers are trained by the obtained bispectrum from vibration datasets recorded in the experiments. The rest of the layers are identically transferred from AlexNet.

Therefore, the whole process of cavitation detection proposed in this study can be presented by the flowchart shown in Figure 1. The vibration signals, recorded from the test setup of a centrifugal pump in cavitation and no-cavitation states, are analyzed using bispectral analysis. The obtained bispectrum images are resized and normalized to fit the input size of the input layer of the CNN. The trained CNN classifies the bispectrum images to "cavitation" or "nocavitation" condition.

\section{Experiments}

The performance of the proposed system is examined through experiments. The tests are conducted on a Gunt test setup at the machinery condition monitoring laboratory of Shahid Chamran University of Ahvaz. The test stand is shown in Figure 2. The studied pump is part of a closed water circuit including input and output valves and hoses, water tank, and connectors. An A/127/V DJB accelerometer is installed on the pump cover using special glue. The accelerometer is connected to $\mathrm{B} \& \mathrm{~K}$ pulse data acquisition system, which records the data at a constant sampling rate of $16 \mathrm{kHz}$.

The water circuit is checked for any air or bubble trapped in the valves or pipes before starting the motor. The input and output valves are kept fully open. Then, at slow rotating speeds, the remaining air is ejected from a nipple valve on the pump cover. After making sure that the water circuit is free of air, the motor speed is increased to $2900 \mathrm{rpm}$ recommended by the vendor. The data of the "no-cavitation" condition are recorded first. Then, we gradually close the input valve until the cavitation bubbles are observed through the transparent casing of the pump. The data related to the "low cavitation" state is acquired when it is half closed, and the data of the "developed cavitation" are recorded when it was almost closed. In Figure 3(a)-3(c), three images of the inside liquid of the pump are provided to show the working conditions of the pump in each state. Under the low- and developed-cavitation conditions, the bubbles around the inlet of the pump are visible as a white cloud.

\section{Numerical Results}

In this section, the classification results using AlexNet and GoogleNet for cavitation detection and then cavitation severity detection are provided.

The recorded signals under each working condition are divided into three parts for training, validating, and testing of the CNN. Each part is divided into small windows comprised of 7000 sample points. In order to augment the training data, the windows have overlaps of 300 sample points for the no-cavitation condition and 1500 sample points for the cavitation condition. No overlap was used for the validation and testing datasets.

In the next step, the bispectrum images were extracted from the generated short segments of signals. One sample bispectrum for each no-cavitation and developed-cavitation states is shown in Figure 4. These are just samples of a variety of patterns obtained from bispectral analysis. In a number of cases, relating the bispectrum to one of the states is not as easy as what is seen in Figure 4, and the patterns from different conditions look similar to each other. Therefore, machine learning should be used for higher accuracies.

In Matlab, the obtained spectrograms are saved and then given to AlexNet, which was prepared in Section 2.2 earlier. 1880 images were used for training. 143 and 385 images were considered for validation and testing, respectively. The size of the input image has to be 227 by 227 by 3 , and the images are resized and normalized before using in the AlexNet. The initial size of the bispectrum as RGB images is 875 by 656 by 3. A minibatch of 30 images was used with 2 epochs. Out of the three available optimizers, SGDM (stochastic gradient descent with momentum) is used because of its better performance. The loss function of cross-entropy loss is also applied.

The accuracy and loss functions are plotted during the training process and are shown in Figure 5. Both the training and validation accuracies reach $100 \%$, and the test accuracy is $100 \%$, as well. There are a number of fluctuations; however, the accuracies reach $100 \%$ eventually.

In the next step, the possibility of detecting the cavitation level is investigated. Again, the recorded signals in each working condition are divided into three parts for training, validating, and testing of CNNs. Each part is divided into small windows comprised of 21000 sample points. In order to augment the training data, the windows have overlaps of 300 sample points for the "no-cavitation condition and 1500 sample points for the low-cavitation and developed-cavitation condition, respectively. No overlap was used for the validation and testing datasets.

Again, Matlab is used for processing the obtained spectrograms. Two pretrained deep neural networks, GoogleNet and AlexNet, are used in order to examine the possibility of obtaining better performance. The AlexNet has all the pretrained layers except for the last three fully connected layers, which are replaced by an 80-neuron fully connected one and a three-class classification output. GoogleNet layers are the same as the pretrained network trained by ImageNet dataset, except for the classification layer, which is replaced by a three-class one. The size of the input image for GoogleNet has to be 224 by 
TABLE 1: The structure of the CNN applied in this research.

\begin{tabular}{lccc}
\hline Stage & Layer no. & Layer type & Size \\
\hline 1 & 1 & Input bispectrum images & $227 \times 227 \times 3$ \\
\hline & 2 & Convolutional & $55 \times 55 \times 96$ \\
2 (transferred) & 3 & ReLU & $55 \times 55 \times 96$ \\
& 4 & Cross-channel normalization & $55 \times 55 \times 96$ \\
& 5 & Max pooling & $27 \times 27 \times 96$ \\
\hline & 6 & Convolutional & $27 \times 27 \times 256$ \\
3 (transferred) & 7 & ReLU & $27 \times 27 \times 256$ \\
& 8 & Cross-channel normalization & $13 \times 13 \times 256$ \\
\hline 4 (transferred) & 9 & Max pooling & $13 \times 13 \times 384$ \\
& 10 & Convolutional & $13 \times 13 \times 384$ \\
5 (transferred) & 11 & ReLU & $13 \times 13 \times 384$ \\
& 12 & Convolutional & $13 \times 13 \times 384$ \\
6 (transferred) & 13 & ReLU & $13 \times 13 \times 256$ \\
& 14 & Convolutional & $13 \times 13 \times 256$ \\
7 (trained) & 15 & ReLU & $6 \times 6 \times 256$ \\
\hline \multirow{2}{*}{8 (trained) } & 16 & Max pooling & 10 \\
& 17 & Fully connected & 10 \\
\hline
\end{tabular}

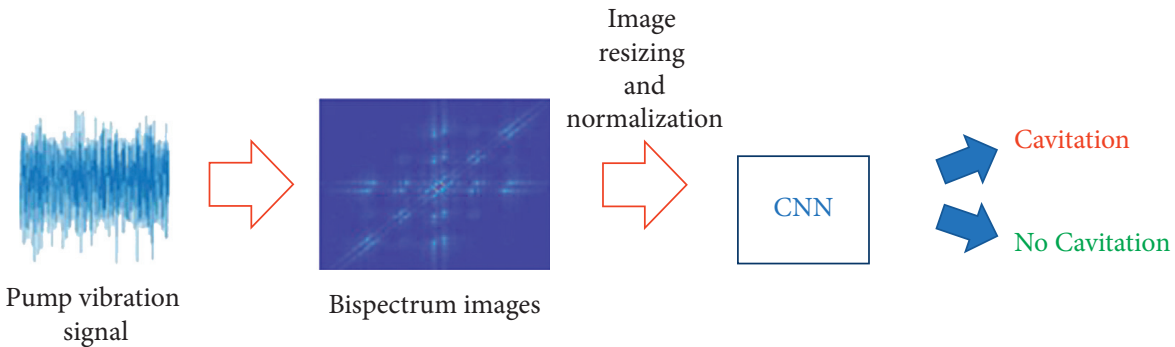

FIgURE 1: The flowchart of the proposed system.

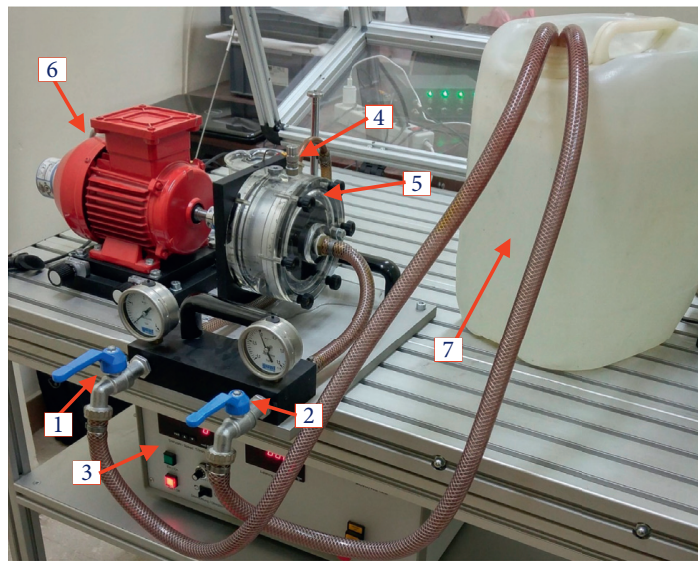

Figure 2: The test setup for pump cavitation studies: (1) output valve, (2) input valve, (3) the box of the motor speed controller, (4) accelerometer, (5) centrifugal pump, (6) electromotor, and (7) water tank.

224 by 3 , and the images are resized and normalized before using in both GoogleNet and AlexNet. A minibatch of 30 images was used with 4 and 5 epochs for AlexNet and GoogleNet, respectively. Adam (adaptive momentum optimizer) with variable learning rates is used because of its better performance for the new networks. The loss function of crossentropy loss is applied again.

The accuracy and loss functions are plotted during the training processes for these two networks and are shown in Figure 6 and 7. A validation accuracy of $95.2 \%$ and the test 


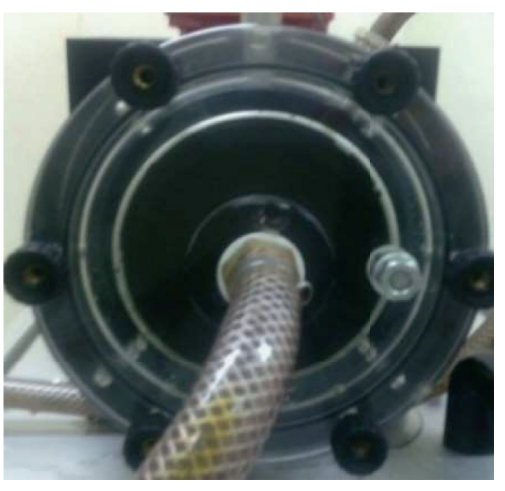

(a)

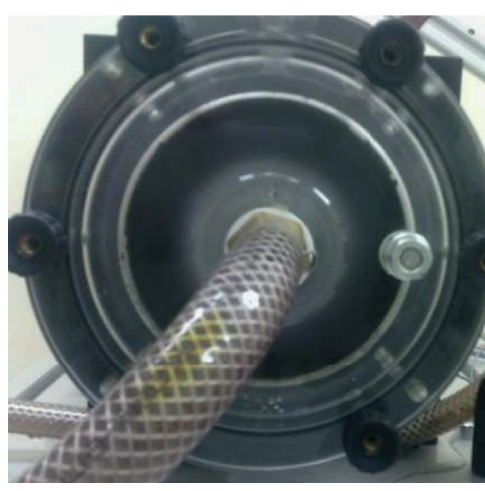

(b)

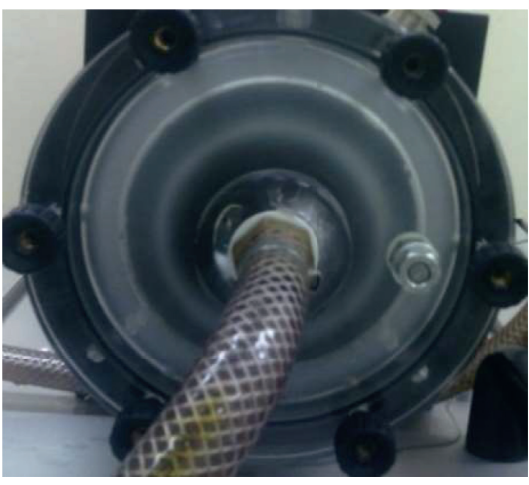

(c)

FIGURE 3: The interior image of the pump under (a) normal (no cavitation), (b) low-cavitation, and (c) developed-cavitation conditions. The cavitation bubbles are seen as white foggy area on the transparent casing of the pump.

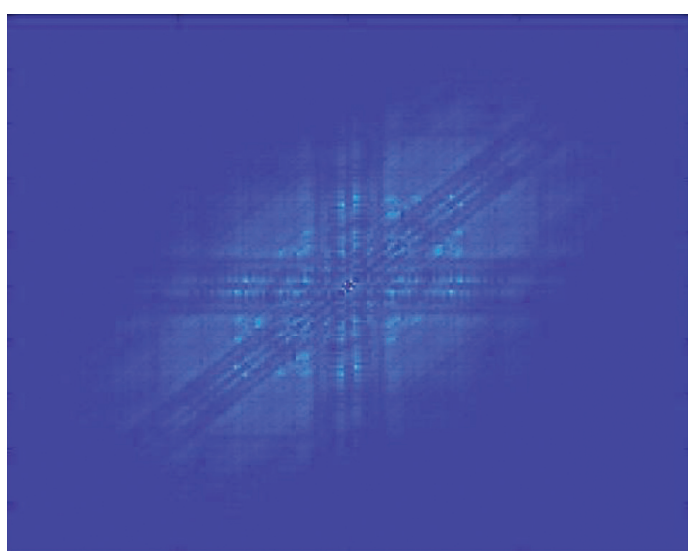

(a)

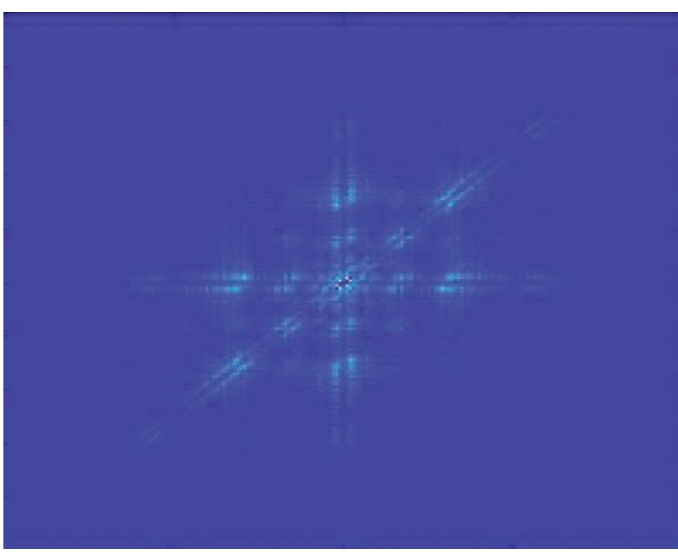

(b)

FIGURE 4: The bispectrum images obtained from (a) "developed-cavitation" state and (b) "no-cavitation" state.

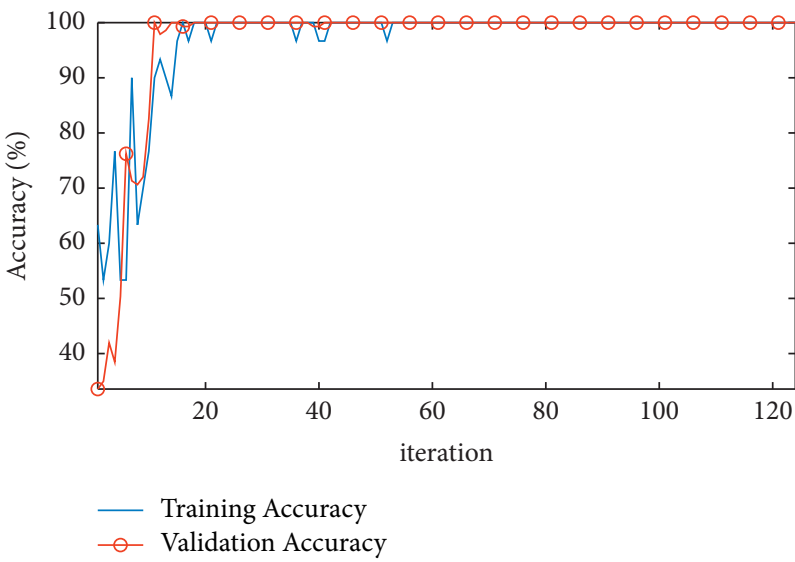

(a)

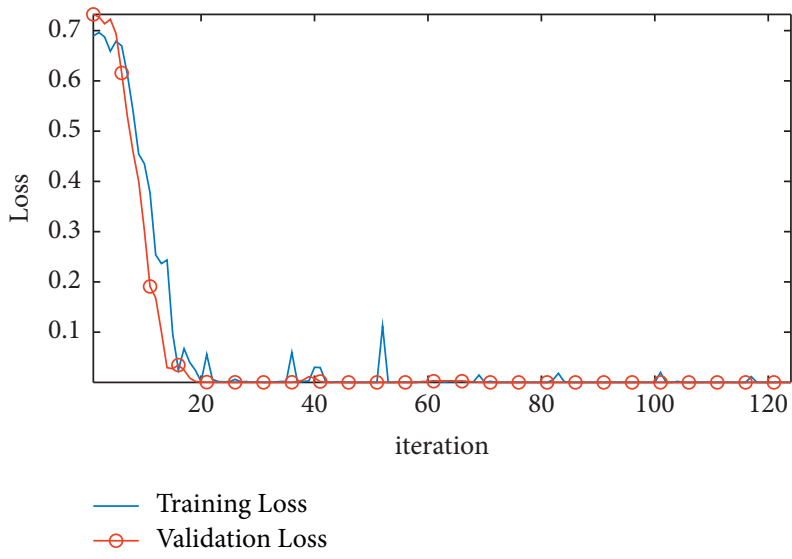

(b)

Figure 5: The training progress of the AlexNet prepared for cavitation detection: (a) accuracy diagram and (b) loss diagram.

accuracy of $98.9 \%$ are reached for the AlexNet. Based on Figure 7 for GoogleNet, a validation accuracy of $93.3 \%$, and from the test data, a test accuracy of $95.1 \%$ are obtained. The training accuracy reaches $100 \%$ in both cases.
By investigating the results of AlexNet, we can show more information in a confusion matrix. The confusion matrix of the intensity detection task of the AlexNet is shown in Figure 8 . Only $4.3 \%$ of the "no-cavitation" test data is 


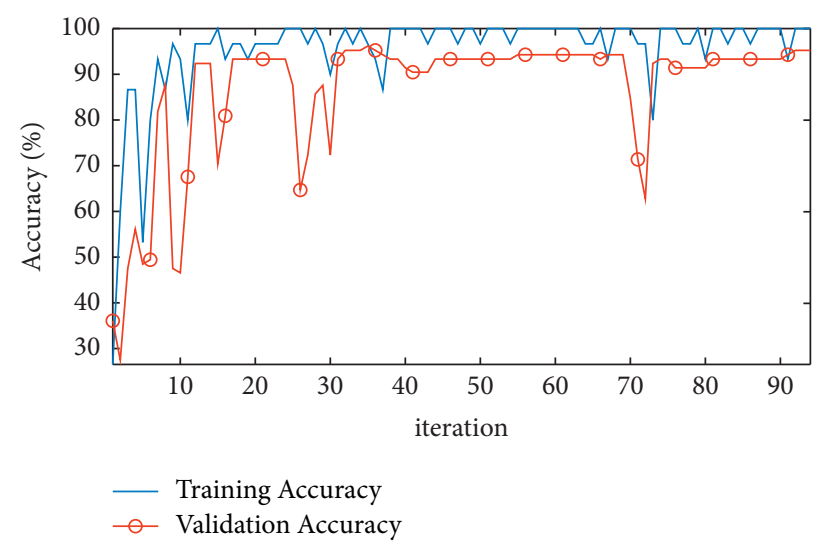

(a)

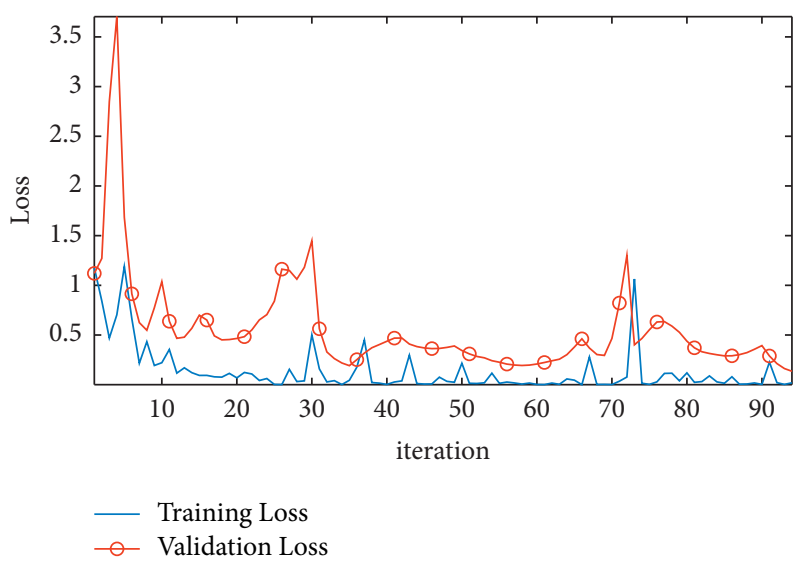

(b)

Figure 6: The training progress of the AlexNet prepared for cavitation severity detection. (a) Accuracy diagram. (b) Loss diagram.

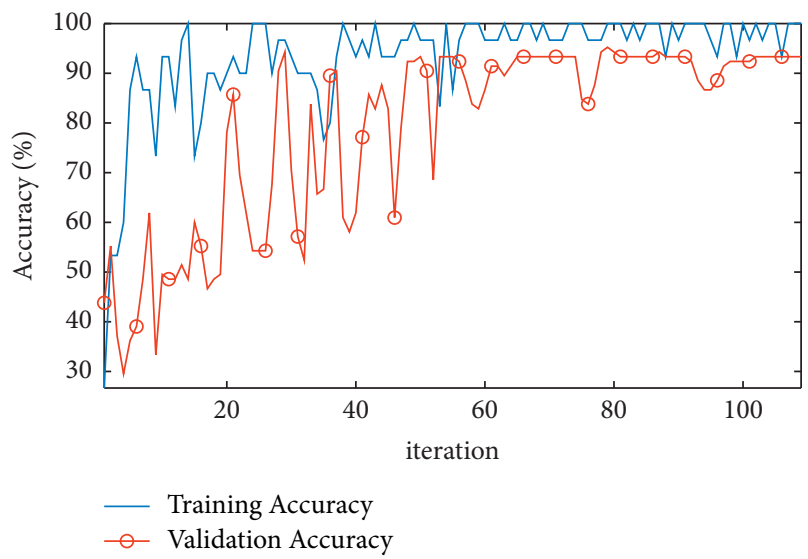

(a)

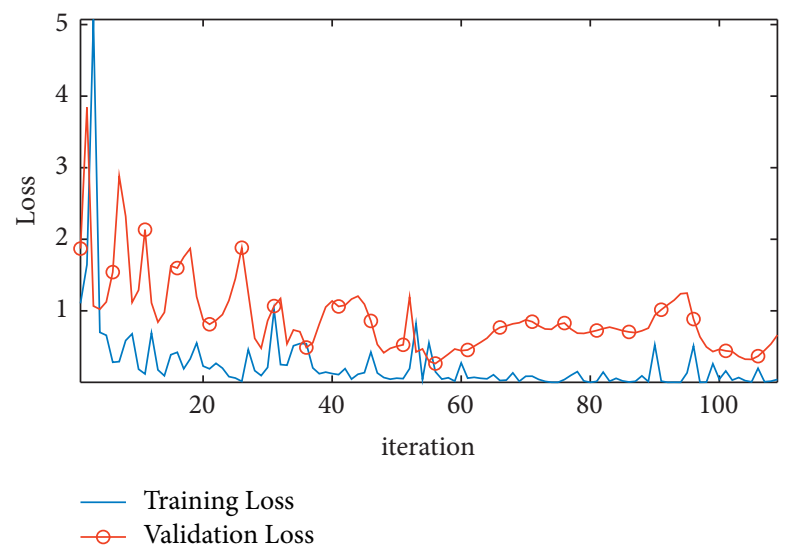

(b)

FIgURE 7: The training progress of the GoogleNet prepared for cavitation severity detection. (a) Accuracy diagram. (b) Loss diagram.

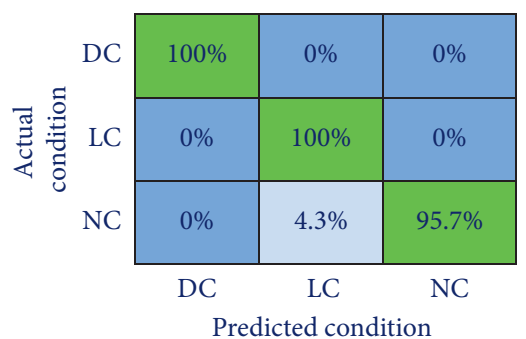

Figure 8: The confusion matrix of the AlexNet for cavitation intensity detection (NC, LC, and DC stand for "no cavitation," "low cavitation," and "developed cavitation," respectively).

predicted as "low cavitation." Other working conditions are correctly predicted.

The confusion matrix shows that even without extracting any explicit features in a separate analysis from the vibration signals, the CNNs have been able to find and calculate distinctive features in their architectures so that they can classify the studied cases of cavitation severity. In conventional diagnosis systems, a feature selection algorithm was also necessary. Therefore, a cavitation analysis system has been developed with less difficulties and by using more complicated inputs.

\section{Conclusions}

In this research work, the vibrations of a centrifugal pump under different cavitation conditions were recorded and processed using bispectral analysis. The obtained bispectrum was generated from the vibration signals and saved as RGB 
images. Pretrained AlexNets were used for the classification of the bispectrum images. Transfer learning made it possible to use the CNNs pretrained on a set of images for other similar tasks with a faster training process. In the next step, the architecture of the AlexNet was modified to suit this problem. Then, it was trained and used to detect cavitation in the pump. After that, with a minor change, it was trained and used for detecting the level of cavitation. A GoogleNet was also trained and used in this problem. Unlike the conventional machine learning methods, no feature was extracted directly, and no feature selection method was applied. The results showed that cavitation was successfully detected without any error in the first step, where the AlexNet was used for cavitation detection. In detecting the cavitation level, both AlexNet and GoogleNet showed promising results. However, the modified AlexNet showed more accurate classification results than the adjusted GoogleNet. The results show that applying bispectrum images as inputs to these two types of pretrained CNNs can accurately diagnose cavitation conditions of centrifugal pumps without any need for image processing, feature extraction, and feature selection. This is especially helpful in cases where limited data are available.

\section{Data Availability}

The vibration data used to support the findings of this study have been deposited in the ResearchGate website repository (https://www.researchgate.net/profile/Ali-Hajnayeb/publications) in "Research items; Data".

\section{Conflicts of Interest}

The author declares no conflicts of interest in preparing this manuscript.

\section{Acknowledgments}

This research was financially supported by Shahid Chamran University of Ahvaz.

\section{References}

[1] A. M. Abdulaziz and A. Kotb, "Detection of pump cavitation by vibration signature," Australian Journal of Mechanical Engineering, vol. 15, no. 2, pp. 103-110, 2017.

[2] A. R. Al-Obaidi and H. Towsyfyan, "An experimental study on vibration signatures for detecting incipient cavitation in centrifugal pumps based on envelope spectrum analysis," Journal of Applied Fluid Mechanics, vol. 12, no. 6, pp. 20572067, 2019.

[3] A. R. Al-Obaidi, "Investigation of effect of pump rotational speed on performance and detection of cavitation within a centrifugal pump using vibration analysis," Heliyon, vol. 5, no. 6, Article ID e01910, 2019.

[4] A. R. Al-Obaidi, "Experimental comparative investigations to evaluate cavitation conditions within a centrifugal pump based on vibration and acoustic analyses techniques," Archives of Acoustics, vol. 45, pp. 541-556, 2020.

[5] A. R. Al-Obaidi, "Detection of cavitation phenomenon within a centrifugal pump based on vibration analysis technique in both time and frequency domains," Experimental Techniques, vol. 44, no. 3, pp. 329-347, 2020.

[6] Y. Li, G. Feng, X. Li, Q. Si, and Z. Zhu, "An experimental study on the cavitation vibration characteristics of a centrifugal pump at normal flow rate," Journal of Mechanical Science and Technology, vol. 32, no. 10, pp. 4711-4720, 2018.

[7] R. Azizi, B. Attaran, A. Hajnayeb, A. Ghanbarzadeh, and M. Changizian, "Improving accuracy of cavitation severity detection in centrifugal pumps using a hybrid feature selection technique," Measurement, vol. 108, pp. 9-17, 2017.

[8] N. Dutta, U. Subramaniam, P. Sanjeevikumar, S. C. Bharadwaj, Z. Leonowicz, and J. B. Holm-Nielsen, "Comparative study of cavitation problem detection in pumping system using SVM and K-nearest neighbour method," in Proceedings of the 2020 IEEE International Conference on Environment and Electrical Engineering and 2020 IEEE Industrial and Commercial Power Systems Europe (EEEIC/IઐCPS Europe), pp. 1-6, IEEE, Madrid, Spain, June 2020.

[9] N. Dutta, S. Umashankar, V. A. Shankar, S. Padmanaban, Z. Leonowicz, and P. Wheeler, "Centrifugal pump cavitation detection using machine learning algorithm technique," in Proceedings of the 2018 IEEE International Conference on Environment and Electrical Engineering and 2018 IEEE Industrial and Commercial Power Systems Europe (EEEIC/ IঊCPS Europe), pp. 1-6, IEEE, Palermo, Italy, June 2018.

[10] A. Hajnayeb, R. Azizi, A. Ghanbarzadeh, and M. Changizian, "Vibration-based cavitation detection in centrifugal pumps," Diagnostyka, vol. 18, pp. 77-83, 2017.

[11] A. K. Panda, J. S. Rapur, and R. Tiwari, "Prediction of flow blockages and impending cavitation in centrifugal pumps using Support Vector Machine (SVM) algorithms based on vibration measurements," Measurement, vol. 130, pp. 44-56, 2018.

[12] M. B. Abadi, A. Hajnayeb, A. Hosseingholizadeh, and A. Ghasemloonia, "Single and multiple misfire detection in internal combustion engines using vold-kalman filter ordertracking," SAE technical paper. 2011:01-1536, SAE, Warrendale, PA, USA, 2011.

[13] Z. Ahmad, A. Rai, A. S. Maliuk, and J.-M. Kim, "Discriminant feature extraction for centrifugal pump fault diagnosis," Ieee Access, vol. 8, Article ID 165512, 2020.

[14] J. Dai, J. Tang, S. Huang, and Y. Wang, "Signal-based intelligent hydraulic fault diagnosis methods: review and prospects," Chinese Journal of Mechanical Engineering, vol. 32, pp. 1-22, 2019.

[15] A. Hajnayeb, K. H. Shirazi, and R. Aghaamiri, "Vibration measurement for crack and rub detection in rotors," $M e$ trology and Measurement Systems, vol. 27, 2020.

[16] G. C. Brito, R. D. Machado, and A. C. Neto, "Model-based vibration condition monitoring for fault detection and diagnostics in large hydrogenerators,"vol. 2, pp. 105-119, in Proceedings of the 10th International Conference on Rotor Dynamics, vol. 2, Springer, Rio de Janeiro, Brazil, September 2018.

[17] M. R. Fatehi, A. Ghanbarzadeh, S. Moradi, and A. Hajnayeb, "Global sensitivity analysis improvement of rotor-bearing system based on the Genetic Based Latine Hypercube Sampling (GBLHS) method," Structural Engineering \& Mechanics, vol. 68, pp. 549-561, 2018.

[18] Z. Gao, C. Cecati, and S. X. Ding, "A survey of fault diagnosis and fault-tolerant techniques-part I: fault Diagnosis with model-based and signal-based approaches," IEEE 
Transactions on Industrial Electronics, vol. 62, no. 6, pp. 3757-3767, 2015.

[19] A. Hajnayeb, J. S. Fernando, and Q. Sun, "Effects of vehicle driveline parameters and clutch judder on gearbox vibrations," Proceedings of the Institution of Mechanical Engineers Part D: Journal of Automobile Engineering, vol. 236, Article ID 09544070211015939, 2021.

[20] X. Bi, S. Cao, and D. Zhang, "Diesel engine valve clearance fault diagnosis based on improved variational mode decomposition and bispectrum," Energies, vol. 12, no. 4, p. 661, 2019.

[21] C. Grover and N. Turk, “A novel fault diagnostic system for rolling element bearings using deep transfer learning on bispectrum contour maps," Engineering Science and Technology, an International Journal, 2021.

[22] J. Guo, D. Zhen, H. Li, Z. Shi, F. Gu, and A. D. Ball, "Fault detection for planetary gearbox based on an enhanced average filter and modulation signal bispectrum analysis," ISA Transactions, vol. 101, pp. 408-420, 2020.

[23] S. Guoji, S. McLaughlin, X. Yongcheng, and P. White, "Theoretical and experimental analysis of bispectrum of vibration signals for fault diagnosis of gears," Mechanical Systems and Signal Processing, vol. 43, no. 1-2, pp. 76-89, 2014.

[24] O. Hamomd, S. Alabied, Y. Xu, A. Daraz, F. Gu, and A. Ball, "Vibration based centrifugal pump fault diagnosis based on modulation signal bispectrum analysis," in Proceedings of the 2017 23rd International Conference on Automation and Computing (ICAC), pp. 1-5, IEEE, Huddersfield, UK, September 2017.

[25] H. Li, Z. Tian, H. Yu, and B. Xu, "Fault prognosis of hydraulic pump based on bispectrum entropy and deep belief network," Measurement Science Review, vol. 19, no. 5, pp. 195-203, 2019.

[26] M. Sandhya, K. Rajarajeswari, and P. Seetaramaiah, "Detecting inception of hydrodynamic cavitation noise of ships using quadratic phase coupling index as an indicator," Defence Science Journal, vol. 65, 2015.

[27] M. Nasiri, M. Mahjoob, and H. Vahid-Alizadeh, "Vibration signature analysis for detecting cavitation in centrifugal pumps using neural networks," in Proceedings of the 2011 IEEE International Conference on Mechatronics, pp. 632-635, IEEE, Istanbul, Turkey, April 2011.

[28] B.-S. Yang, W.-W. Hwang, D.-J. Kim, and A. Chit Tan, "Condition classification of small reciprocating compressor for refrigerators using artificial neural networks and support vector machines," Mechanical Systems and Signal Processing, vol. 19, no. 2, pp. 371-390, 2005.

[29] A. Hajnayeb, A. Ghasemloonia, S. E. Khadem, and M. H. Moradi, "Application and comparison of an ANNbased feature selection method and the genetic algorithm in gearbox fault diagnosis," Expert Systems with Applications, vol. 38, no. 8, pp. 10205-10209, 2011.

[30] V. Sugumaran, D. Jain, M. Amarnath, and H. Kumar, "Fault diagnosis of helical gear box using decision tree through vibration signals," International Journal of Performability Engineering, vol. 9, p. 221, 2013.

[31] M. Boumahdi, J.-P. Dron, S. Rechak, and O. Cousinard, "On the extraction of rules in the identification of bearing defects in rotating machinery using decision tree," Expert Systems with Applications, vol. 37, no. 8, pp. 5887-5894, 2010.

[32] A. Hajnayeb, S. Khadem, and M. Moradi, "Design and implementation of an automatic condition-monitoring expert system for ball-bearing fault detection," Industrial Lubrication \& Tribology, vol. 60, pp. 93-100, 2008.
[33] A. Sharma, V. Sugumaran, and S. Babu Devasenapati, "Misfire detection in an IC engine using vibration signal and decision tree algorithms," Measurement, vol. 50, pp. 370-380, 2014.

[34] L. S. Dhamande and M. B. Chaudhari, "Detection of combined gear-bearing fault in single stage spur gear box using artificial neural network," Procedia Engineering, vol. 144, pp. 759-766, 2016.

[35] M. Cerrada, R. Sánchez, D. Cabrera, G. Zurita, and C. Li, "Multi-stage feature selection by using genetic algorithms for fault diagnosis in gearboxes based on vibration signal," Sensors, vol. 15, no. 9, Article ID 23903, 2015.

[36] X. Yu, F. Dong, E. Ding, S. Wu, and C. Fan, "Rolling bearing fault diagnosis using modified LFDA and EMD with sensitive feature selection," Ieee Access, vol. 6, pp. 3715-3730, 2017.

[37] S. Guo, T. Yang, W. Gao, and C. Zhang, "A novel fault diagnosis method for rotating machinery based on a convolutional neural network," Sensors, vol. 18, no. 5, p. 1429, 2018.

[38] M. T. Pham, J.-M. Kim, and C. H. Kim, “Accurate bearing fault diagnosis under variable shaft speed using convolutional neural networks and vibration spectrogram," Applied Sciences, vol. 10, no. 18, p. 6385, 2020.

[39] Z. Chen and W. Li, "Multisensor feature fusion for bearing fault diagnosis using sparse autoencoder and deep belief network," IEEE Transactions on Instrumentation and Measurement, vol. 66, no. 7, pp. 1693-1702, 2017.

[40] W. Qian, S. Li, P. Yi, and K. Zhang, "A novel transfer learning method for robust fault diagnosis of rotating machines under variable working conditions," Measurement, vol. 138, pp. 514-525, 2019.

[41] S. S. Udmale, S. K. Singh, R. Singh, and A. K. Sangaiah, "Multi-fault bearing classification using sensors and ConvNet-based transfer learning approach," IEEE Sensors Journal, vol. 20, pp. 1433-1444, 2019.

[42] F. Dong, X. Yu, X. Shi, K. Liu, Z. Wu, and W. Yu, "A new transferable fault diagnosis approach of rotating machinery based on deep autoencoder and dominant features selection under different operating conditions," Shock and Vibration, vol. 2021, Article ID 7383255, 21 pages, 2021.

[43] L. Saidi, "The deterministic bispectrum of coupled harmonic random signals and its application to rotor faults diagnosis considering noise immunity," Applied Acoustics, vol. 122, pp. 72-87, 2017.

[44] X. Pang, X. Xue, W. Jiang, and K. Lu, “An investigation into fault diagnosis of planetary gearboxes using a bispectrum convolutional neural network," IEEE/ASME Transactions on Mechatronics, vol. 26, 2020. 